\title{
Charge Density Based Small Signal Modeling for InSb/AllnSb Asymmetric Double Gate Silicon Substrate HEMT for High Frequency Applications
}

T. VENISH KUMAR ( $\nabla$ tvenishkumar@gmail.com )

Sethu Institute of Technology https://orcid.org/0000-0002-9292-8994

\section{Venkatesh}

REVA University

B. Muthupandian

Sethu Institute of Technology

G. Lakshmi Priya

VIT University - Chennai Campus

\section{Research Article}

Keywords: Indium Antimonide, TCAD, Double Gate HEMT, Semiconductor, Capacitance

Posted Date: August 18th, 2021

DOI: https://doi.org/10.21203/rs.3.rs-804003/v1

License: (c) (i) This work is licensed under a Creative Commons Attribution 4.0 International License.

Read Full License 


\title{
Charge Density based Small Signal Modeling for InSb/AlInSb Asymmetric Double Gate Silicon Substrate HEMT for High Frequency Applications
}

\author{
T.Venishkumar ${ }^{*}$, M.Venkatesh $^{2}$, B.Muthupandian ${ }^{3}$, G.Lakshmi Priya $^{4}$ \\ ${ }^{1}$ Department of Electronics and Communication Engineering, Sethu Institute of Technology, \\ tvenishkumar@gmail.com \\ ${ }^{2}$ School of Electronics and Communication Engineering, \\ REVA University, Bengaluru-560064, Karnataka, India \\ venkateshmm92@gmail.com \\ ${ }^{3}$ Department of Electronics and Communication Engineering, Sethu Institute of Technology, \\ mpspmuthu09@gmail.com \\ ${ }^{4}$ School of Electronics Engineering (SENSE), VIT University, Chennai-600127,TamilNadu \\ lakshmipriya.g@vit.ac.in
}

\begin{abstract}
The Asymmetric Double Gate Silicon Substrate HEMT(ADG-Si-HEMT) is proposed in this article to analyse the carrier concentration and intrinsic small signal parameters for the heterostructure of the InSb/AIInSb silicon wafer DG-HEMT device. When the top and bottom gates are biased with different gate voltages, the HEMTs act as a three-port system and the device called Asymmetric Double Gate HEMT. The modulation of back-channel charge density due to the front gate voltage is analyzed with the position of quasi-Fermi energy levels( $\left.E_{f}\right)$. Also, the small signal model is obtained for various parameters like cut off frequency, gate to source capacitance and transconductance. The effects of the following parameters like delta doping, width of Silicon doping layer and various top and bottom gate voltages are analyzed to get enhanced device operation. The transconductance $2390 \mathrm{Sm} / \mathrm{mm}$ for $V_{f g}=0.2 \mathrm{~V}$ and cut off frequency around $197 \mathrm{GHz}$ for $V_{b g}=0.3$ are obtained. All the analytical results are compared with Sentaurus 3-D TCAD simulation results. The asymmetric biasing technique offers various mixed application due to modulation in threshold voltage and modulating carrier density in dual channels. Keywords: Indium Antimonide; TCAD; Double Gate HEMT; Semiconductor; Capacitance

\section{Introduction}

The Indium Antimonide ( $\mathrm{InSb}$ ) Silicon substrate based High Electron Mobility Transistors (HEMTs) are the most suitable for high-speed applications due to their highest electron mobility $\left(30,000 \mathrm{~cm}^{2} \mathrm{~V}^{-1} \mathrm{~S}^{-1}\right)$ and high saturation velocity $\left(5 \times 10^{7} \mathrm{~cm} / \mathrm{s}\right)$ than the other known compound semiconductors [1-2]. In this article,InSb based asymmetric HEMTs are presented to enhance the performance of the HEMT. When the top and bottom gates are biased with the same and different voltages, the device called symmetric and asymmetric HEMTs respectively [3-4].
\end{abstract}


In the Asymmetric Silicon wafer DG-HEMT, the potential well or 2DEG is formed in the direction perpendicular to the interface of $\mathrm{AlInSb}$ and InSb layers because of its bandgap difference and ionized electrons are transferred from $\delta$ doped layer to InSblayer [5]. The transferred electrons are accumulated in the narrow bandgap material ( $\mathrm{InSb}$ ), so it is called potential well or two-dimensional electron gas (2DEG) [6]. The Coulomb scattering is reduced in the high electron mobility transistor because the electrons are separated from the donor atoms. The electrons are permitted to move in the vertical direction of an interface and the concentration of electrons depends on the gate bias and delta doping of the barrier layer [7-8].

The operation of Asymmetric Double Gate HEMT differs from the typical HEMTs. The bottom channel carrier concentration is fixed by the back gate voltage in the fabrication itself and variable voltage is applied to the top gate. Therefore, the top gate took control and modulates the electrons accumulation in both channels. This system works like typical HEMTs but the single gate controls the dual channel and it provides double the amount of current density than the conventional HEMTs. The variable front gate bias provides better immunity against the second order effects, enhanced gate control over the channel and additional flexibility achieved in the threshold voltage by controlling the backchannel carrier concentration [9].

Therefore, a detailed analysis is necessary to understand the sheet charge density $\left(\mathrm{n}_{\mathrm{s}}\right.$ $\mathrm{Cm}^{-2}$ ) in the Asymmetric DG-HEMTdevice. There are several linear and nonlinear analytical models are proposed since the 1980s to calculate charge density in the channel. The linear approximation for Fermi-level $\left(\mathrm{E}_{\mathrm{f}}\right)$ versus sheet charge density $\left(\mathrm{n}_{\mathrm{s}}\right)$ done by Drummond and team[10]. Since 1988, various other research groups are expressed the non-linear approximation of $E_{f}$ versus $n_{s}$ but these approximations are appropriate only if devices are not operated in the subthreshold region [11]. Further, Dasgupta and his expresses the good nonlinear approximation but this expression is not suitable for a wider range of $n_{s}$ values [12].

Finally, the new expression for Fermi energy level versus sheet charge density is proposed by Jinrong and Fermi energy level $\left(E_{f}\right)$ is calculated for three different values of ns in the year 2019 [13-14]. Based on this approximation the Asymmetric double gate HEMT sheet charge density (ns) is calculated for three different regions of operation $\left(V_{\mathrm{fg}}>\mathrm{V}_{\text {th } 1}+\mathrm{k}_{1}, \mathrm{~V}_{\mathrm{th} 1}<\mathrm{V}_{\mathrm{fg}}<\right.$ $\left.\mathrm{V}_{\mathrm{th} 1}+\mathrm{k}_{1},<\mathrm{V}_{\mathrm{th} 1}+\mathrm{k}_{1}\right)$. Further, the front gate modulation on the backchannel is expressed using Gauss's law. In general, this analysis leads to further analysis of the frequency performance and speed of carrier in the channel. Because the gate length and gate foot to channel distance are interrelated to the frequency performance of the device [15-17]. So, In the continuous analysis of charge density, the work extended to calculate the small-signal model to extract the Gate to Source Capacitance, Cut off Frequency and Transconductance are obtained of Asymmetric DGHEMT.

\section{Device Structure Description}

Fig.1 shows the cross-sectional view of Asymmetric Double Gate HEMTs (DGHEMTs). The wide bandgap material AluminiumIndium Antimonide(AlInSb) grown over a narrow bandgap material Indium Antimonide( $\mathrm{InSb})$, therefore the 2-dimensional electron gas or quantum well is formed in narrow bandgap material, unlike the MOSFETs. In the Asymmetric DG-HEMT device, two quantum wells are formed on the back and forth of the InSb layer because the InSb is inserted between the two wide bandgaps AlInSb materials and two identical gates are placed on the top $\left(\mathrm{V}_{\mathrm{fg}}\right)$ and bottom $\left(\mathrm{V}_{\mathrm{bg}}\right)$ of the device structure. The cap layer is used to reduce the conduct resistance of ohmic conducts, followed by AlInSb barrier layer width is $\mathrm{T}_{\mathrm{a}}$, AlInSb spacer width is $T_{s}$, delta doping AlInSb layer width is $T_{d}$ with doping concentration $N_{D}$ 
and the total width of AlInSb layer is $\mathrm{T}=\mathrm{T}_{\mathrm{a}}+\mathrm{T}_{\mathrm{s}}+\mathrm{T}_{\mathrm{d}}$ on both sides of InSb layer thickness is $\mathrm{T}_{\mathrm{ch}}$. The device parameters are listed in Table. 1

Table 1 Device dimensions

\begin{tabular}{|c|c|c|c|}
\hline S.No. & Layers & Doping & Dimensions (nm) \\
\hline 1 & Cap layer & $5 \times 10^{25} \mathrm{~cm}-3$ & 10 \\
\hline 2 & Silicon substrate Doped AlInSb & $2 \times 10^{23} \mathrm{~cm}-3$ & 3 \\
\hline 3 & Te $\delta-$ doping $\left(\mathrm{T}_{\mathrm{a}}\right)$ & $3 \times 10^{23} \mathrm{~cm}-3$ & 7 \\
\hline 4 & Silicon wafer AlInSb $\left(\mathrm{T}_{\mathrm{s}}\right)$ & $1 \times 10^{18} \mathrm{~cm}-3$ & 20 \\
\hline 5 & Silicon wafer InSb $\left(\mathrm{T}_{\mathrm{ch}}\right)$ & - & 80 \\
\hline 6 & Gate Length $(\mathrm{L})$ & - & \\
\hline
\end{tabular}

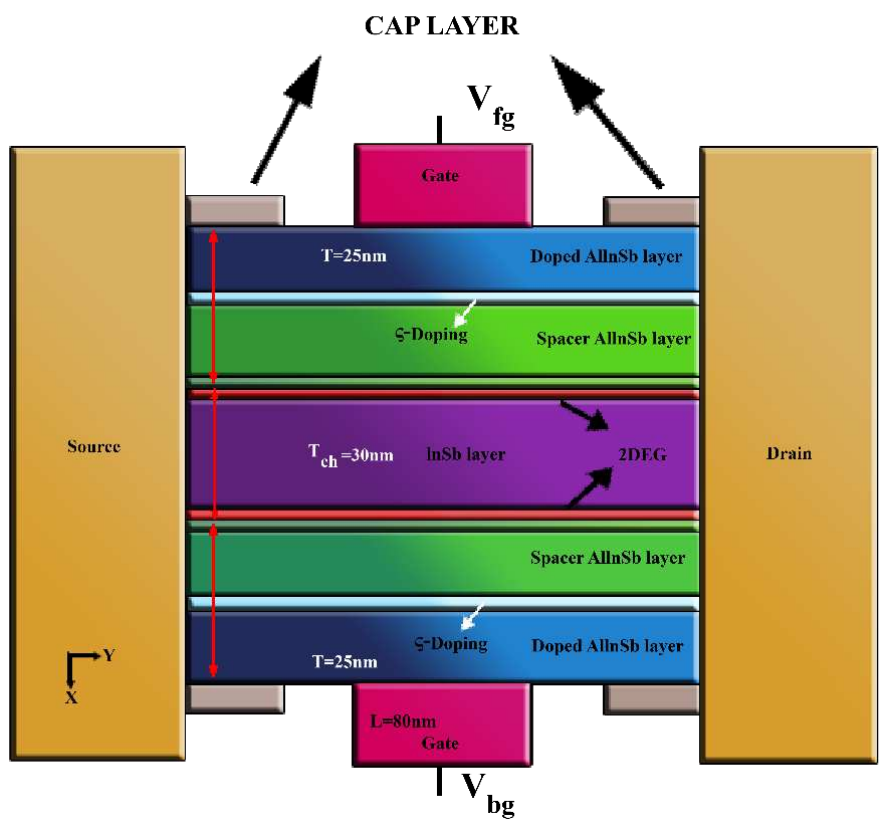

Fig.1 Cross sectional view of InSb/AlInSb Silicon Substrate Asymmetric DG-HEMTs 


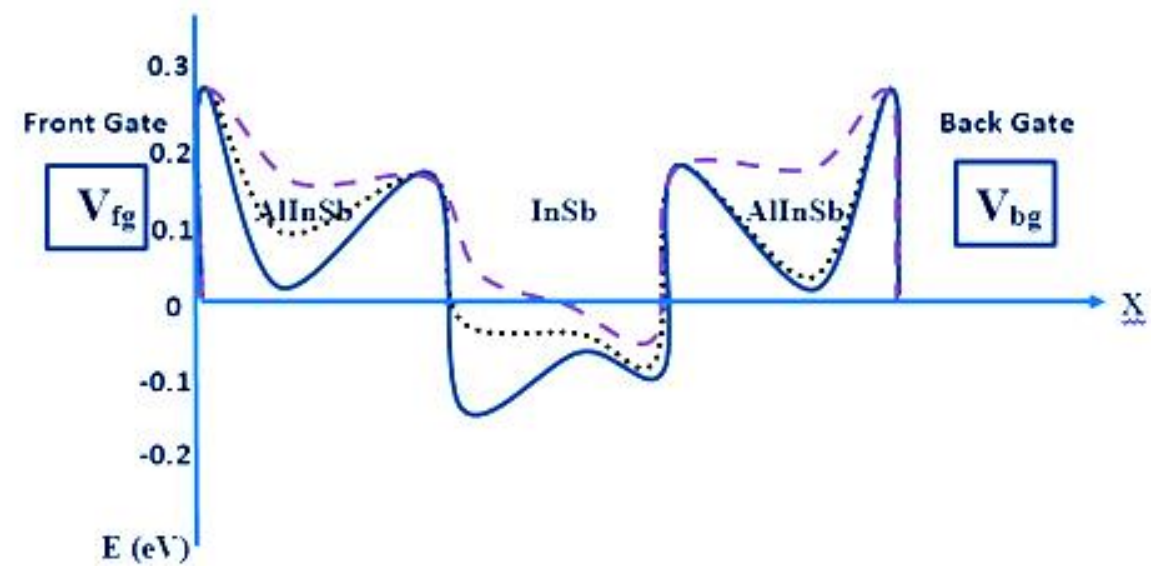

Fig.2 Energy band diagram of InSb/AIInSb Asymmetric Silicon wafer DG-HEMT

Fig.2 shows the energy band diagram of the Asymmetric DG-HEMT for three different circumstances. The solid line signifies the conduction of both top and bottom quantum well, the dotted line signifies the front channel is in the threshold region but the backchannel is conducting, the dashed line signifies the front channel is in the cut off regions but the backchannel is conducting with fixed back gate voltage $\mathrm{V}_{\mathrm{bg}}$. In this analytical model, the source and drain contacts are limited for channel region and do not consider for parallel conduction.

\section{Charge Density Model for Asymmetric Silicon wafer DG-HEMT}

\subsection{Top and Bottom 2-Dimensional Electron Gas}

The 2-dimensional electron gas/quantum well is formed at the boundary of two dissimilar compound semiconductor due to the variance in the electron affinity of the materials. The electron transfer across the interface can be written by equating the depletion of carriers from the barrier AlInSb Silicon layer to the accumulation of carriers in the quantum well InSb Silicon layer. The maximum quantity of carriers is depleted from the AlInSb barrier layer is given by using Lee theory [18],

$$
n_{s d i}=\sqrt{\frac{2 \varepsilon_{A} N_{D i}}{q}\left(\Delta E_{c i}-E_{F i}\right)+N_{D i}{ }^{2} T_{s i}{ }^{2}}-N_{D i} T_{s i}
$$

where $\varepsilon_{\mathrm{A}}$ is the permittivity of the AlInSb barrier layer, $\Delta \mathrm{E}_{\mathrm{ci}}$ is the conduction band discontinuity of top ( $\mathrm{i}=1)$ and bottom ( $\mathrm{i}=2$ ) hetero-interface, $\mathrm{E}_{\mathrm{Fi}}$ quasi-fermi energy level of top and bottom hetero-interface and $\mathrm{q}$ is the electron charge.

For large negative voltage is applied to the gates, the gate depletion and the heterointerface depletion will overlap, in this case,equation (1) is replaced by the Poisson Equation for the solution of top and bottom interface and expressed by

$$
n_{s i}=\frac{\varepsilon_{A}}{q T}\left(V_{G S i}-V_{o f f i}-E_{F i}\right)
$$

where, $\mathrm{V}_{\mathrm{GSi}}\left(\mathrm{i}=1\right.$ for top-gate bias $\mathrm{V}_{\mathrm{fg}}$ and $\mathrm{i}=2$ for bottom gate bias $\left(\mathrm{V}_{\mathrm{bg}}\right), \mathrm{V}_{\text {off }}$ is threshold voltage. 


$$
V_{o f f i}=\phi_{b i}-\Delta E_{c i}-\frac{q N_{D} T_{\delta}^{2}}{2 \varepsilon_{A}}
$$

where $\phi_{\text {bi }}$ barrier height of the top and bottom gate.

The model formulated using the relationship among the sheet charge density $\left(\mathrm{n}_{\mathrm{s}}\right)$ and quasi-Fermi energy level $\left(\mathrm{E}_{\mathrm{F}}\right)$ and the accurate model for Fermi level $\left(\mathrm{E}_{\mathrm{F}}\right)$ versus carrier concentration $\left(\mathrm{n}_{\mathrm{s}}\right)$ is expressed by $\mathrm{Pu}[19]$.

$$
E_{F i}=K_{1}+\left(K_{2} n_{s i}+K_{3} n_{s i}^{2}\right)^{\frac{1}{2}}
$$

where, $\mathrm{K}_{1}, \mathrm{~K}_{2}, \mathrm{~K}_{3}$ are temperature-dependent parameters.

\subsection{Sheet charge density of top heterointerface AlInSb/InSb $\left(\mathbf{n}_{\mathbf{s} 1}\right)$}

The charge density of the top channel is found by substitute equation (3) and (4) in

$$
\begin{aligned}
& n_{s 1}=\frac{\varepsilon_{A}}{q T}\left(V_{f g}-\phi_{b i}-\Delta E_{c 1}-\frac{q N_{D} T_{\delta}^{2}}{2 \varepsilon_{A}}-K_{1}-\left(K_{2} n_{s 1}+K_{3} n_{s 1}{ }^{2}\right)^{\frac{1}{2}}\right) \\
& n_{s 1} \frac{q T}{\varepsilon_{A}}+\phi_{b i}+\Delta E_{c 1}+K_{1}-V_{f g}+\frac{q N_{D} T_{\delta}^{2}}{2 \varepsilon_{A}}=-\left(K_{2} n_{s 1}+K_{3} n_{s 1}{ }^{2}\right)^{\frac{1}{2}}
\end{aligned}
$$

The top channel sheet charge density $\left(\mathrm{n}_{\mathrm{s} 1}\right)$ is obtained as

$$
n_{s 1}=\frac{-B+\sqrt{B^{2}-4 A C}}{2 A}
$$

where,

$$
\begin{aligned}
& A=\left(\frac{q T}{\varepsilon_{A}}\right)^{2}-K_{3} \\
& B=2 \frac{q T}{\varepsilon_{A}}\left(\phi_{b i}+\Delta E_{c 1}+K_{1}-V_{f g}+\frac{q N_{D} T_{\delta}^{2}}{2 \varepsilon_{A}}\right)-K_{2} \\
& C=\left(\phi_{b i}+\Delta E_{c 1}+K_{1}-V_{f g}+\frac{q N_{D} T_{\delta}^{2}}{2 \varepsilon_{A}}\right)^{2}
\end{aligned}
$$

\subsection{Sheet charge density of bottom heterointerface $\operatorname{InSb/AIInSb}\left(n_{\mathbf{s} 2}\right)$}

Similarly, the backchannel carrier concentration is fixed by the back gate bias $\left(\mathrm{V}_{\mathrm{bg}}\right)$, but the large voltage applied to the top gate $\left(\mathrm{V}_{\mathrm{fg}}\right)$ at $\mathrm{V}_{\mathrm{fg}}=\mathrm{V}_{\text {off1 }}$ the top channel enters into the subthreshold region and the carriers are got depleted subsequently at $\mathrm{V}_{\mathrm{fg}}=\mathrm{V}_{\text {off } 1}+\mathrm{K}_{1}$. Therefore, the top gate bias is starting to modulate the back gate channel carrier concentration at $V_{f g}>V_{\text {off1 }}$. The modulation of back-channel carrier concentration $\left(n_{s m}\right)$ is expressed as [20-21] 


$$
n_{s m}=\frac{\varepsilon_{A}}{q T}\left(E_{1}-E_{2}\right)-N_{A} T_{c h}
$$

where the $E_{1}$ and $E_{2}$ are the vertical electric field at the top and bottom interface and is given by

$$
\begin{aligned}
& E_{1}=\frac{V_{f g}-V_{o f f 1}-E_{F 1}}{T} \\
& E_{2}=\frac{V_{b g}-V_{o f f 2}-E_{F 2}}{T}
\end{aligned}
$$

Substituting equation (9) and (10) in equation (8), we get the modulation of the back carrier concentration along with front gate voltageand the fixed bottom gate voltage is formulated as

$$
n_{s m}=\frac{\varepsilon_{A}}{q T}\left(\frac{V_{f g}-V_{o f f 1}-E_{F 1}}{T}-\frac{V_{b g}-V_{o f f 2}-E_{F 2}}{T}\right)-N_{A} T_{c h}
$$

To obtain the charge control model for the modulated backchannel, the new relationship between the quasi-fermi energy level of back-channel $\left(\mathrm{E}_{\mathrm{F} 1}\right)$ and the modulated carrier concentration by front gate bias is required.

$$
n_{s d 2}=n_{s 2}-n_{s m}
$$

The above equation leads to the new relationship between the $\mathrm{E}_{\mathrm{Fd} 2}$ and $\mathrm{n}_{\mathrm{sd} 2}$ same as reported in Equation (4) and is given by

$$
E_{F d 2}=K_{1}+\left(K_{2} n_{s d 2}+K_{3} n_{s d 2}{ }^{2}\right)^{\frac{1}{2}}
$$

However, the depletion of charge carriers in the backchannel is equal to the accumulation of charge carriers in the top heterointerface. So that the top heterointerface is the function of $\mathrm{E}_{\mathrm{Fd} 2}$ and it is formulated as

$$
E_{F 1}=-E_{F d 2}+E_{1} T_{c h}
$$

In the last term in the above equation, the multiplication of top hetero-interface $\left(\mathrm{E}_{1}\right)$ and channel thickness $\left(\mathrm{T}_{\mathrm{ch}}\right)$ is added to analyse the effect of channel thickness in the charge control model.

$$
E_{F 1}=-K_{1}-\left(K_{2} n_{s d 2}+K_{3} n_{s d 2}^{2}\right)^{\frac{1}{2}}+E_{1} T_{c h}
$$

Similar to front channel sheet charge density $\left(n_{s 1}\right)$ andcarrier density $\left(n_{s 2}\right)$ of the backchannel is found from the equation (4) (11) and (15).

The total sheet charge density of Asymmetric DG-HEMTdevice for the three regions of operation is expressed as 


$$
n_{s}=\left\{\begin{array}{c}
n_{s 1}+n_{s 2}, V_{f g}>V_{o f f 1}+K_{1} \\
n_{s 1}+n_{s 2}, V_{o f f 1}<V_{f g}<V_{o f f 1}+K_{1} \\
n_{s 2}, V_{f g} \leq V_{o f f 1}+K_{1}
\end{array}\right.
$$

\subsection{Drain Current Model for AlInSb/InSb/AlInSb Silicon substrate Asymmetric DG- HEMT}

The general solution of the drain current model for Asymmetric DG-HEMT is given by

$$
I_{D}=q W n_{s}(y) v(y)
$$

where the $v(y)$ is the carrier velocity in the perpendicular direction of the heterointerface taken as a field-dependent parameter [22-23] and expressed as

$$
v(y)=\left\{\begin{array}{c}
\frac{\mu_{0} E(y)}{1+\frac{E(y)}{2 E_{c}}} E<2 E_{c} \\
v_{s a t} E \geq 2 E_{c}
\end{array}\right.
$$

where the $\mathrm{E}(\mathrm{y})=\frac{d V(y)}{d y}$ is the electric field in the 2-dimensional electron gas, $\mu_{0}$ is the carrier mobility at the heterointerface, $v_{s a t}$ is the electron saturation velocity at the interface and $\mathrm{E}_{c}=$ $\frac{v_{s a t}}{\mu_{0}}$ is the critical field.

\subsection{Front channel drain current (ID1) at the linear region $\left(V_{d}<V_{\text {sat }}\right)$}

The drain current for the top channel at the linear region is found by substituting the equation (18) in general drain current model Equation (17) for $v(y)$ at $E<2 E_{c}$.

$$
\begin{aligned}
& I_{D 1}=q W n_{S}(y) \frac{\mu_{0} E(y)}{1+\frac{E(y)}{2 E_{c}}} \\
& I_{D 1}\left[1+\frac{d V(y)}{2 E_{c} d y}\right]=q W n_{s}(y) \mu_{0} \frac{d V(y)}{d y}
\end{aligned}
$$

Taking integration on both sides with respect y from " 0 " to " $L$ " and substituting the boundary conditions $V(0)=0$ and $V(L)=v_{d}$, we obtain the drain current of the top channel as

$$
I_{D 1}=\frac{w q \mu_{0} f\left(x_{1}\right)-f\left(x_{0}\right)}{2 A\left(L+\frac{\mu_{0} v_{d}}{2 v_{s a t}}\right)}
$$

where

$$
\begin{aligned}
& f(x)=-B x+\frac{2 x^{\frac{3}{2}}}{3} \\
& x_{1}=\left(\frac{q T}{\varepsilon_{A}}\right)^{2}-K_{3}+2 \frac{q T}{\varepsilon_{A}}\left(\phi_{b i}+\Delta E_{c 1}+K_{1}-V_{f g}+v_{d}+\frac{q N_{D} T_{\delta}^{2}}{2 \varepsilon_{A}}\right)-K_{2}
\end{aligned}
$$




$$
x_{2}=\left(\frac{q T}{\varepsilon_{A}}\right)^{2}-K_{3}+2 \frac{q T}{\varepsilon_{A}}\left(\phi_{b i}+\Delta E_{c 1}+K_{1}-V_{f g}+\frac{q N_{D} T_{\delta}^{2}}{2 \varepsilon_{A}}\right)-K_{2}
$$

\subsection{Front channel drain current (ID1) at saturation $\left(V_{d} \geq V_{\text {sat }}\right)$}

The drain current is obtained for the saturation region at the channel near the drain, the electron velocity is saturated at $\mathrm{v}_{\text {sat }}$.

$$
I_{\text {sat } 1}=\frac{w q v_{s a t}\left(-B+2 x^{1 / 2}\right)}{2 A}
$$

where,

$x=\left(\frac{q T}{\varepsilon_{A}}\right)^{2}-K_{3}+2 \frac{q T}{\varepsilon_{A}}\left(\phi_{b i}+\Delta E_{c 1}+K_{1}-V_{f g}+v_{s a t}+\frac{q N_{D} \delta_{\delta}^{2}}{2 \varepsilon_{A}}\right)-K_{2}$

$v_{d}=v_{\text {sat }}$ at the channel near the drain.

\subsection{Back-channel drain current (ID2)}

The expression of the drain current for backchannel heterointerface $\left(\mathrm{I}_{\mathrm{D} 2}\right)$ requires the sheet charge density $\left(\mathrm{n}_{\mathrm{sm}}\right)$ (variation effect of back-channel due to the front gate voltage) at the backchannel but it is complicated to solve equation (15) and (4) iteratively. So that the sheet charge density $\left(n_{\mathrm{sm}}\right)$ is obtained using the polynomial relationship between the modulated carrier densities $\left(\mathrm{n}_{\mathrm{sm}}\right)$ and front gate bias $\mathrm{V}_{\mathrm{fg}}[21]$.

The drain current for backchannel heterointerface is obtained using a fourth-order polynomial relationship.

$$
\mathrm{n}_{s m}=\sum_{j=1}^{4} p_{j}\left[V_{f g}-V(y)\right]^{j}
$$

where, $p_{j}$ is the polynomial coefficient.

Substituting the equation (23) and (18) in (17) we obtain the drain current expression for backchannel heterointerface for $v(y)$ at $E<2 E_{c}$ as follows

$$
I_{D 2}=w q \sum_{j=0}^{4} p_{j}\left[V_{f g}{ }^{i+1}-\left(V_{f g}-V(y)\right)^{i+1}\right] \frac{\mu_{0} E(y)}{1+\frac{E(y)}{2 E_{c}}}
$$

Integrating the above equation concerningfor to y from " 0 " to " $\mathrm{"}$ " and $V(0)=$ 0 and $V(L)=V_{D S}$, the drain current expressed as

For linear region

$$
I_{D 2}=\frac{w q \mu_{0}}{\left(L+\frac{\mu_{0} v_{d}}{2 v_{s a t}}\right)} \sum_{j=0}^{4} p_{j}\left[V_{f g}^{j+1}-\left(V_{f g}-V_{D S}\right)^{j+1}\right]
$$

For Saturation region 


$$
I_{\text {sat } 2}=\frac{w q \mu_{0}}{\left(L+\frac{\mu_{0} v_{d}}{2 v_{s a t}}\right)} \sum_{j=0}^{4} \frac{p_{j}}{j+1}\left[V_{f g}{ }^{j+1}-\left(V_{f g}-V_{D S a t 2}\right)^{j+1}\right]
$$

Finally, the total drain current is obtained from the top and bottom heterointerface drain current equations (21) to (25) and is express as

$$
I_{D}=\left\{\begin{array}{c}
I_{D 1}+I_{D 2}, V_{f g}>V_{o f f 1}+K_{1} \\
I_{D 1}+I_{D 2}, V_{o f f 1}<V_{f g}<V_{o f f 1}+K_{1} \\
I_{D 2}, V_{f g} \leq V_{o f f 1}+K_{1}
\end{array}\right.
$$

\subsection{Small Signal Equivalent Circuit}

The traditional small signal equivalent circuit is shown in the Fig.3, it consists only of the intrinsic components. $\mathrm{R}_{\mathrm{i} 1}, \mathrm{R}_{\mathrm{i} 2}, \mathrm{R}_{\mathrm{gd}}, \mathrm{C}_{\mathrm{gd} 1}, \mathrm{C}_{\mathrm{gd} 2}, \mathrm{C}_{\mathrm{gs} 1}, \mathrm{C}_{\mathrm{gs} 2}$, are the intrinsic parasitic resistance and capacitance. The ratio between the variation in the drain current with respect to changes in the drain to source voltage called drain current $\left(\mathrm{G}_{\mathrm{d}}\right)$.

$$
G_{d}=\left.\frac{d I d s}{d V d s}\right|_{V g s=\text { Constant }}
$$

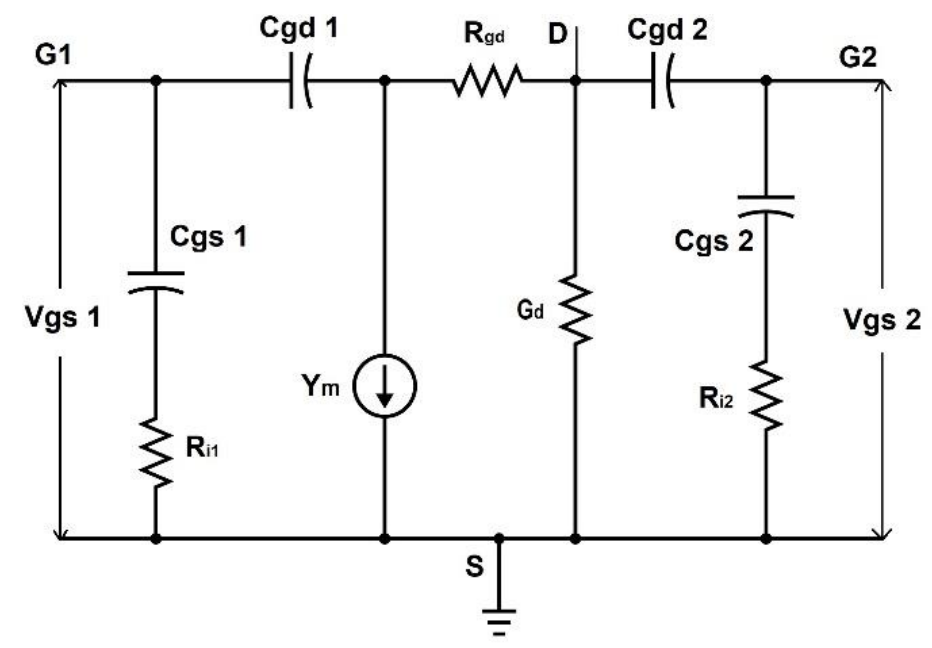

Fig.3 Small Signal Circuit for Asymmetric Silicon substrate DG-HEMTs

The $Y_{m}$ is calculated using the following equation

$$
\mathrm{Ym}=g_{m} \mathrm{e}^{-\mathrm{i} \omega \tau} V_{g s i}
$$

where $\mathrm{Vgs} 1=\mathrm{V}_{\mathrm{fg}}, \mathrm{Vgs} 2=\mathrm{V}_{\mathrm{bg}}, \tau$ - transient time, $\omega$ - angular frequency.

$\mathrm{g}_{\mathrm{m}}$ is the transconductance and it is calculated as the ratio of drain current to gate to source voltage with constant drain voltage. 


\section{Results and Discussions}

In this sheet chargemodel, the 2-dimensional electron gas concentration of the front and back channel is analyzed with a fixed back gate voltage and variable front gate voltages for three different regions of operation at the position of $\mathrm{x}=0$ and $\mathrm{x}=\mathrm{T}_{\mathrm{ch}}$. Also, the relationship of the backchannel with the applied front gate voltage is expressed using Gauss's Law. The drain current is developed separately for the front and back channel because the independent bias is applied to the gates and the device act as a three-port system. The drain current is compared with sentaurus 3D-TCAD tool simulation results.

Fig.4 (a) and (b) predicts the electron density modulation in the backchannel along with front gate voltage for three different fixed back gate voltages. The negative voltage at the front gate starts to deplete the charge carriers on the backchannel. The continuous injection of increasing negative voltage on the front gate completely depletes all charge carries and makes the backchannel turn off, this modulation effect predicted in equation (11).

Further, the backchannel carrier concentration also depends on the back gate bias, the channel thickness, and doping, it is shown in Fig.4(b). In this plot, both the channel thickness $\left(\mathrm{T}_{\mathrm{ch}}=30 \mathrm{~nm}, 25 \mathrm{~nm}\right)$ is saturated at the same level of the backchannel carrier concentration with fixed back gate bias. But, the thicker channel requires more negative voltage to turn off the device. Also, the thick channel layer creates two separate 2DEG at the top and bottom of the channel layer. The top gate controls both channels but the shrinking in the channel thickness may cause two junctions of the interface to gets overlap. Besides, the channel thickness causes a serious problem in the threshold voltage variation. The proper selection of the channel thickness is yields better performance in the dual-channel technology. If the fixed voltage of the bottom gate is reduced, the carrier concentration of the backchannel get reduces, which also reduces the required front gate voltage to completely deplete charge carriers in the backchannel. This effect predicted in Fig. $4 \mathrm{a}$ and $\mathrm{b}$, the back gate bias at $\mathrm{V}_{\mathrm{bg}}=-0.1 \mathrm{~V}$ requires more negative front gate bias to deplete the charge carriers in the backchannel but it is comparatively low for $\mathrm{V}_{\mathrm{bg}}=-0.2 \mathrm{~V}$ and $\mathrm{V}_{\mathrm{bg}}=-0.3 \mathrm{~V}$.

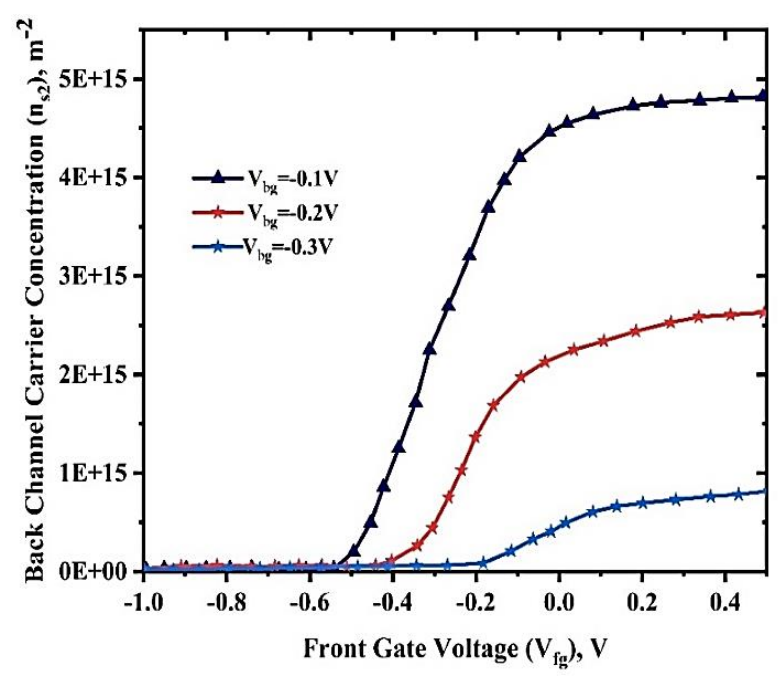

(a)

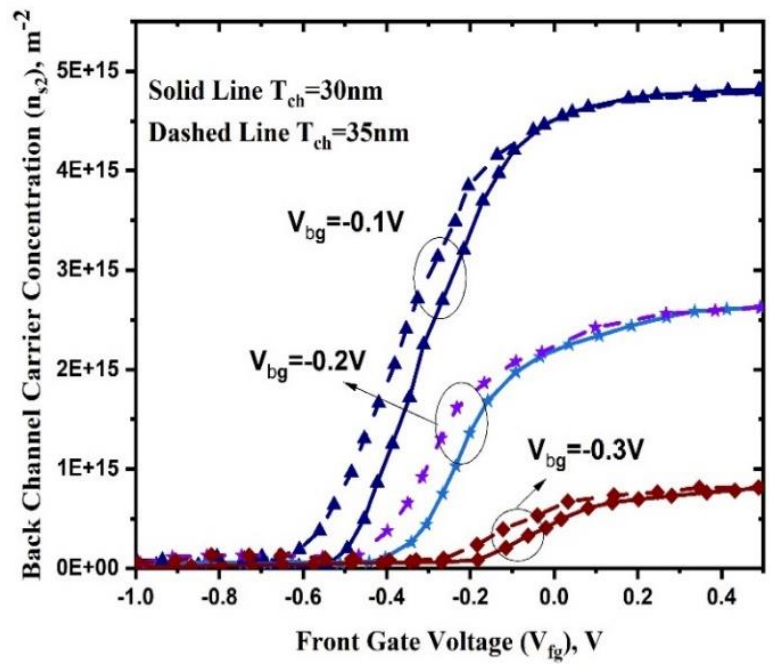

(b)

Fig.4 variation of $n_{s 2}$ with front gate voltage for (a) three different back gate voltage $V_{b g}=-0.1,-0.2,-0.3$ and $(b)$ two different $T_{c h}=30,35$ mmfor different back gate voltage $\left(V_{b g}\right)$ 
The expression (16) indicates that the total charge carrier concentrations of the channel depends on both variable front gate voltage and fixed back gate voltage but the threshold voltage varies concerning the carrier concentration of both channel. Therefore, the backchannel has less threshold voltage than the front channel, for which the back gate bias is fixed less than the variable front gate voltage.

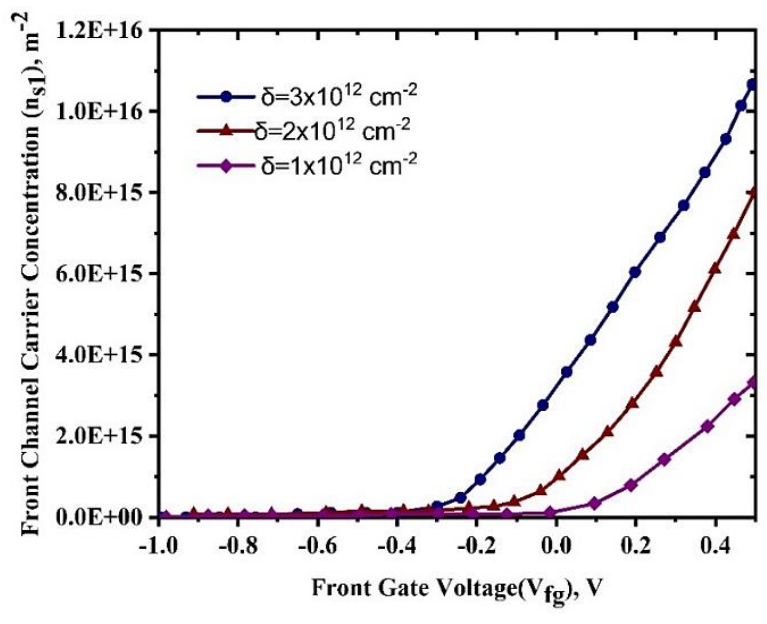

(a)

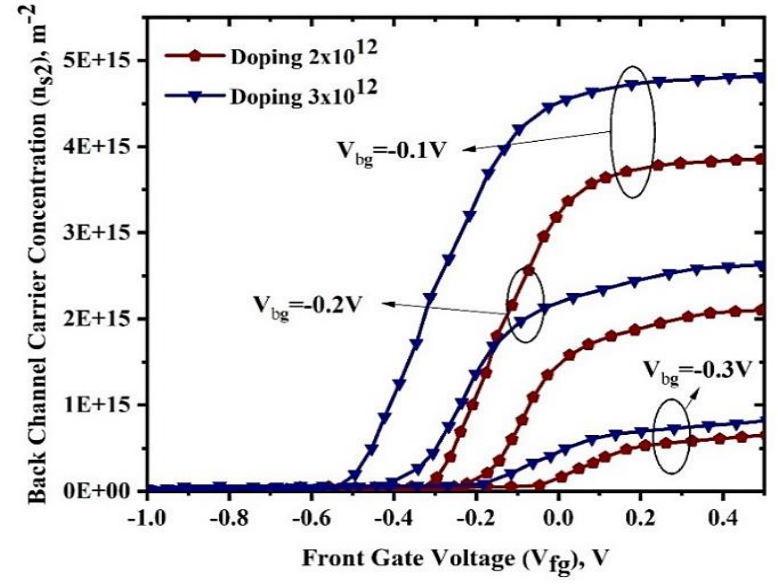

(b)

Fig.5 (a) Variation of front channel density $\left(n_{s 1}\right)$ for 3 different $\delta$ doping, (b) Back channeldensity $\left(n_{s 2}\right)$ compared with two different doping with 3 different $V_{b g}$.

The front channel electron density ns1 for three different $\delta$ doping $\left(3 \times 10^{12}\right.$, $\left.2 \times 10^{12}, 1 \times 10^{12} \mathrm{~cm}^{-2}\right)$ and back channel electron density for two different $\delta$ doping $\left(3 \times 10^{12}\right.$, $\left.2 \times 10^{12} \mathrm{~cm}^{-2}\right)$ with three different back gate voltages $(-0.1,-0.2,-0.3 \mathrm{~V})$ are compared in figure $4.2 \mathrm{a} \& \mathrm{~b}$. Both graphs clearly show that the density of charge carriers in both channels depends on the delta doping concentration of the barrier layer. According to the solution given in equation (14), After the front channel reaches the subthreshold region, the carrier density in the quantum well increases with the accumulation of electrons from the top barrier layer also depleted electrons from the back channel. Therefore, the single gate takes control of both channels in the Asymmetric Silicon substrate DG-HEMTs. Further, the electrons accumulation depends on the barrier width. If the device has a larger barrier width, it increases the distance between the channel and the gate foot causes the reduction in electrons in the quasi-fermi level, this was explained in the previous section. The subthreshold voltage also varies with the channel thickness and the interface charges of 2DEG.

In the Asymmetric DG-HEMTs, the doping is done on both sides of the channel (InSb) with the AlInSb barrier layer to enhance the accumulation of electrons in the quantum well. Therefore, doping profile analysis is an unavoidable important parameter in the density of carrier concentration calculation on both the top and bottom of the two-dimensional electron gas. The top barrier doping profile was explained in Fig.5(a) and the bottom barrier doping profile analysis shown in Fig.5(b). The graph clearly shows the larger delta doping profile with the same back gate voltage produces high carrier density on back channel. Also, the larger doping profile shifts the entire plot to the left side, which indicates that the variation of threshold voltage with delta doping. Further, the electron concentration drop on the channel by decreasing the delta doping in the barrier layer is compensated by surface charge density. 


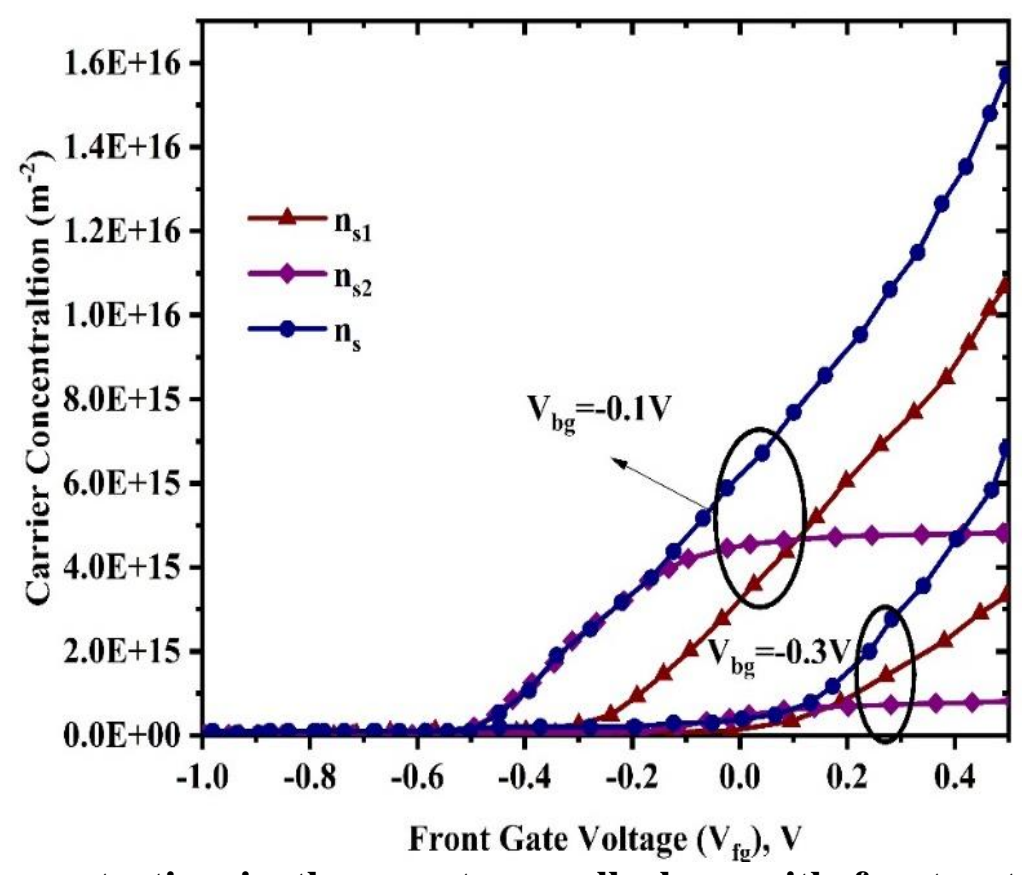

Fig.6 Carrier concentration in the quantum well along with front gate voltage for two different back gate voltages $\left(\mathrm{V}_{\mathrm{bg}}=\mathbf{- 0 . 1} \mathrm{V}, \mathrm{V}_{\mathrm{bg}}=\mathbf{- 0 . 3 \mathrm { V }}\right)$

The charge density $\mathrm{n}_{\mathrm{s} 1}, \mathrm{n}_{\mathrm{s} 2}$ and total charge density $\mathrm{n}_{\mathrm{s}}$ relationships are shown in Fig.6, for two different back-gate voltages $\left(\mathrm{V}_{\mathrm{bg}}=-0.1 \mathrm{~V}\right.$ and $\left.\mathrm{V}_{\mathrm{bg}}=-0.3 \mathrm{~V}\right)$. The graph clearly shows thatthe total carrier density $\mathrm{n}_{\mathrm{s}}$ follows then 2 up to the front channel reaches the subthreshold region of the operation. When the top channel enters into the subthreshold region, the total carrier density $n_{s}$ variation depends on both the top and bottom of carrier concentration $\left(n_{s}\right)$. In addition, the plot shows that the top channel reaches the maximum density and it depletes the backchannel carrier concentration due to modulation effects on the backchannel by front gate bias. But the total carrier density is the function of both the top and bottom carrier density of quantum well. However, the backchannel concentration is the only function of back gate voltage and the density of the charge carriers is saturated and no longer varies for fixed back gate bias. Therefore, the total charge density (ns) variation is only due to the modulation effect in the top channel charge carriers. 


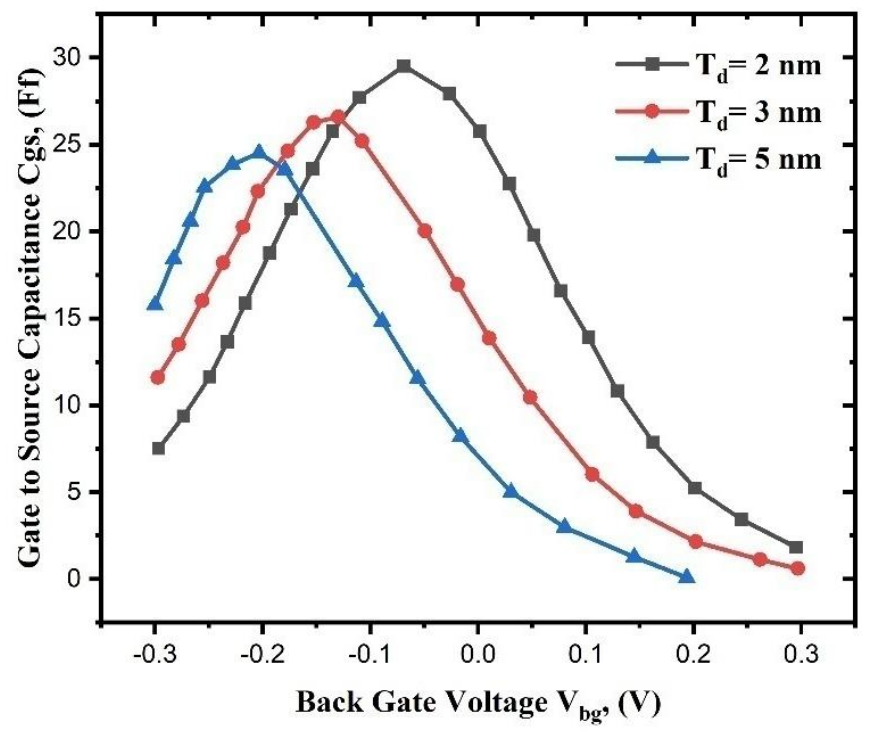

Fig.7 Gate Source Capacitance variation along with back gate voltage for three different channel thickness $T_{d}=2,3,5 n m, V_{d s}=0.3 V$.

The function of donor layer thickness (Td) in the gate to source capacitance along with back gate voltage is shown in Fig.7. The thick donor layer $(5 \mathrm{~nm})$ shows the low gate to source capacitance but it led to more negative voltage needed to deplete the quantum well formulated under the donor layer. As mentioned in Fig.2, the distance between the gate foot and the quantum well is increased, the gate loses control over the channel.

The frequency variation for three different back gate voltage along with front gate voltage $\left(\mathrm{V}_{\mathrm{fg}}\right)$ is shown in Fig.8. The cut offfrequency $(\mathrm{Ft})$ linearly increasing by increasing the biasing voltage on the back gate from negative and reaches the maximum of operation speed and get reduces with increasing front gate biasing voltage. These effects observed because of modulation in the transconductance and gate to source capacitance when increasing voltage in the front gate beyond the limit of saturation.So, understanding of transconductance relationship with front and back gate voltages is an unavoidable analysis and it is shown in Fig.9.

It is observed that the transconductance is increasing along with back gate voltage with constant front gate bias and reaches the maximum, further increasing bias at the back gate it starts to degreases. Also, increasing front gate bias could achieve high total transconductance. Because the front gate controls the total transconductance of the device. 


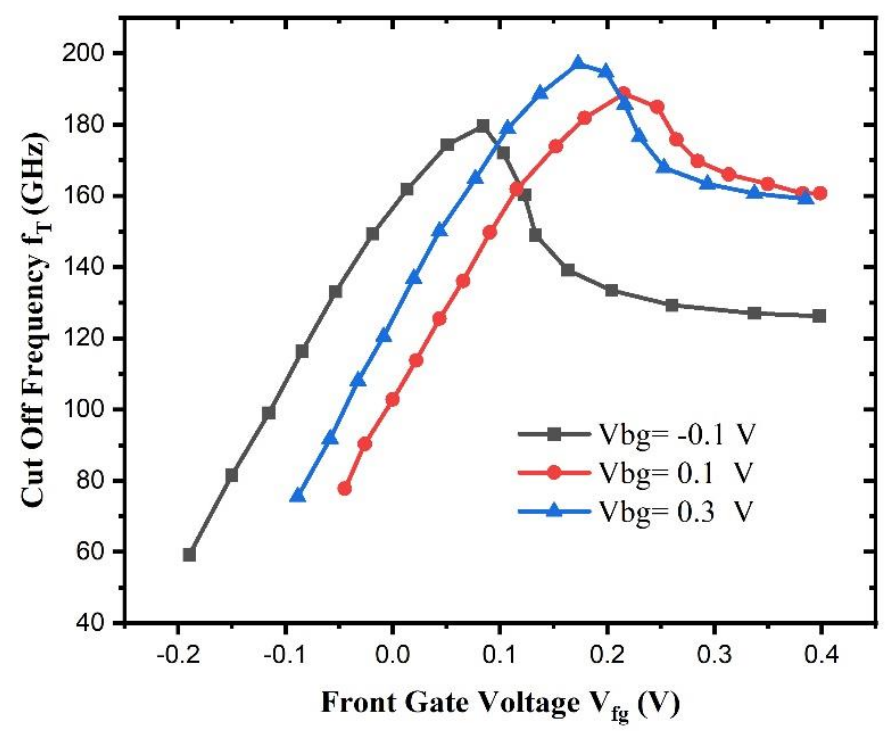

Fig.8 Cut-off frequency variation along with front gate voltage for three different back gate voltage $V_{b g}=-0.1,0.1,0.3 \mathrm{~V}$ at $V_{d s}=0.3 \mathrm{~V}$.

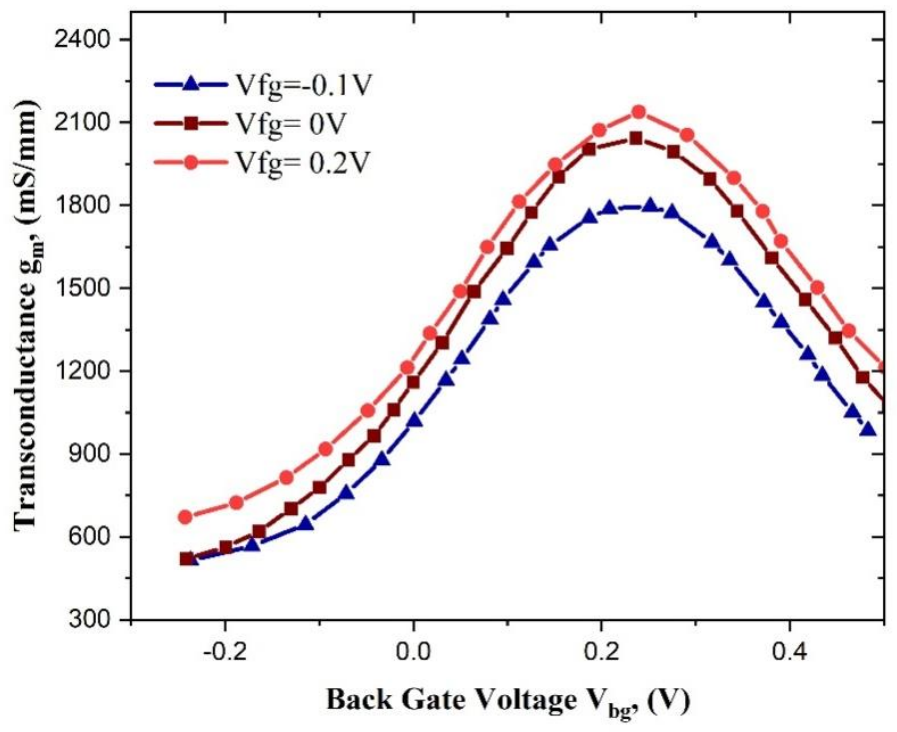

Fig.9. Transconductance $\left(g_{m}\right)$ variation along with back gate voltage for three different front gate voltage $V_{f g}=-0.1,0,2 \mathrm{~V}, V_{d s}=0.3 \mathrm{~V}$.

The drain current for three different back gate bias with front gate voltage is shown in Fig.10. The plot clearly shows that the back gate bias varies the threshold voltage because the backchannel concentration is a function of the back gate voltage. The variable threshold voltage achieved by modulating backchannel carrier concentration with back gate voltage also provides better results against the short channel effects. The backchannel carrier concentration is fixed by 
the fixed back gate voltage during the fabrication. The developed drain current expressions result closely matched with sentaurus TCAD simulation results.

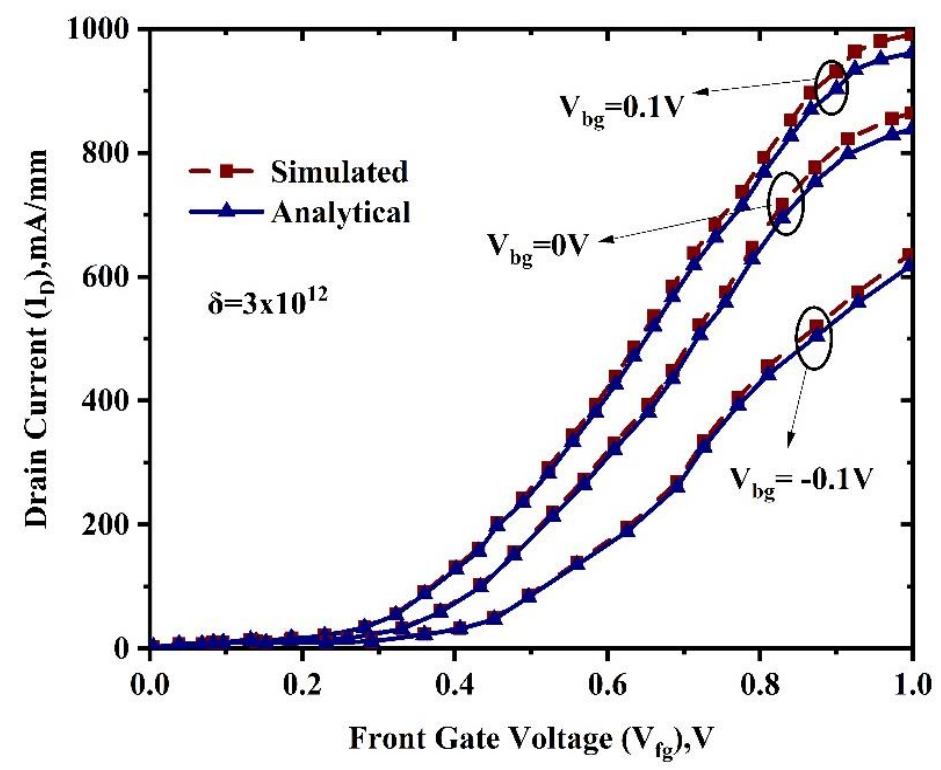

Fig.10 Variation of drain current versus front gate voltage for three different back gatevoltages $\left(\mathrm{V}_{\mathrm{bg}}=0.1 \mathrm{~V}, \mathrm{~V}_{\mathrm{bg}}=0 \mathrm{~V}_{\mathrm{bg}}=\mathbf{- 0 . 1 \mathrm { V }}\right)$

Fig.11 shows the drain current variations versus drain to source voltage for different front gate voltages. The drain current results are developed according to equation (27) by considering both the channels. The carrier concentration of the channel or drain current approaches zero at which the threshold voltage is calculated for the Asymmetric DG-HEMT. The drain current for various front gate bias compared with sentaurus TCAD simulation results.

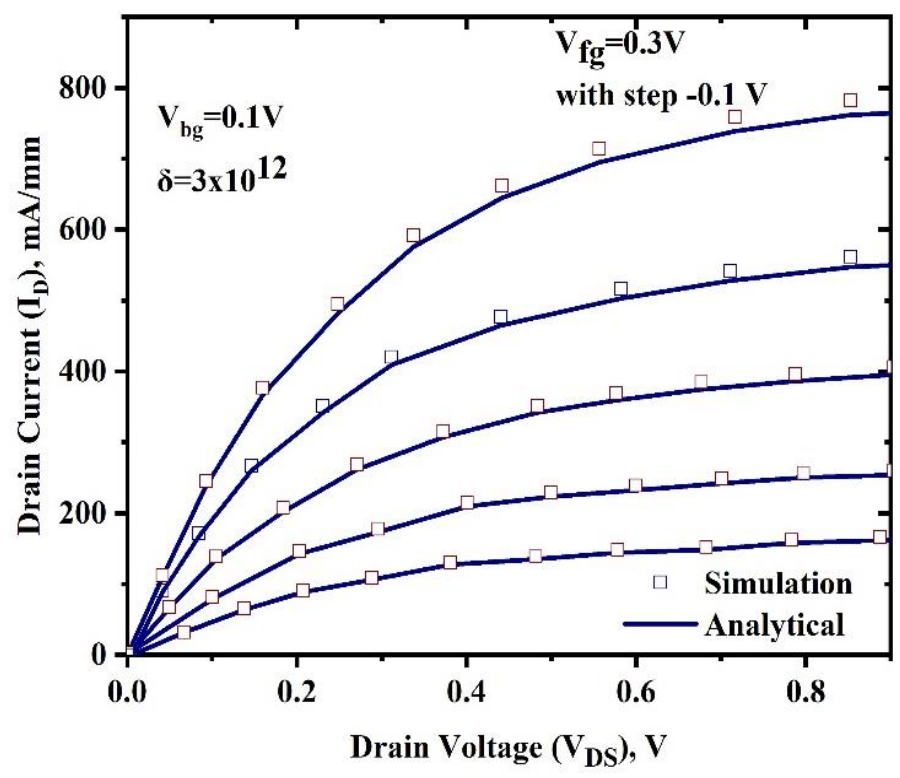

Fig.11 Variation of drain current versus drain voltage for different front gate voltages 


\section{Conclusion}

A compact model has developed to predict the sheet charge density and small signal intrinsic parameters for Asymmetric Silicon substrate DG-HEMT. The sheet charge density $\left(\mathrm{n}_{\mathrm{s}}\right)$ is developed by relating the quasi-fermi energy level and it predicts the modulation effects on the back channel by different front gate biases. The variation of carrier concentration on dual channel also investigated by various parameters like back gate bias, doping concentration, barrier and Silicon channel thickness. The relationship between the threshold voltage variation due to modulation of carrier concentration is studied. The variable threshold voltage devices are used in the mixed-mode circuits for high frequency applications. The proposed small signal model is used to obtain the Gate to Source Capacitance and Cut Off frequency with a function of dual channels and we obtained the Cut Off frequency around $197 \mathrm{GHz}$ for $\mathrm{V}_{\mathrm{bg}}=0.3 \mathrm{~V}$. The transconductance of the dual channel is also obtained around $2390 \mathrm{Sm} / \mathrm{mm}$ for $\mathrm{V}_{\mathrm{fg}}=0.2 \mathrm{~V}$. In additions, the model parameters are extended to develop drain current characteristics of Asymmetric Silicon wafer DG-HEMT. The analytical results of drain current are verified and compared with sentaurus 3-D TCAD results. Simulation results are closely matched with obtained results of the charge control model.

\section{Acknowledgements Not Applicable}

Funding The authors of the manuscript did not receive any funding, grants, or in-kind support in support of the research or the preparation of the manuscript.

Availability of Data and Material There are no linked research data sets for this submission. The following reason is given: No data was used for the research described in the article.

Code Availability - Not Applicable

Author's Contributions Author 1 (T.Venish Kumar): Conceived and design the analysis, contributed data,analysis tools, and wrote the paper. Author 2 (M.Venkatesh): Performed the analysis, calibrated the results, and wrote the paper. Author 3 (B.Muthupandian): Worked in TCAD portion of the proposed device and analytical Modeling. Author 4 (G.Lakshmi Priya): Worked in TCAD simulation of drain current modeling, and wrote the paper for the corresponding portion.

Compliance with ethical standards- Yes

Consent to participate-Yes 


\section{Consent for Publication-Yes}

Conflict of Interest All authors have participated in (a) conception and design, or analysis and interpretation of the data; (b) drafting the article or revising it critically for important intellectual content; and (c) approval of the final version. This manuscript has not been submitted to, nor is under review at, another journal or other publishing venue. The authors have no affiliation with any organization with a direct or indirect financial interest in the subject matter discussed in the manuscript.

\section{$\underline{\text { Authorship Conformation }}$}

All authors have participated in (a) conception and design, or analysis and interpretation of the data; (b) drafting the article or revising it critically for important intellectual content; and (c) approval of the final version.

$>$ This manuscript has not been submitted to, nor is under review at, another journal or other publishing venue.

$>$ The authors have no affiliation with any organization with a direct or indirect financial interest in the subject matter discussed in the manuscript

$>$ The following authors have affiliations with organizations with direct or indirect financial interest in the subject matter discussed in the manuscript:

$\underline{\text { Author's name }}$

1.T.VENISH KUMAR

2.M.VENKATESH

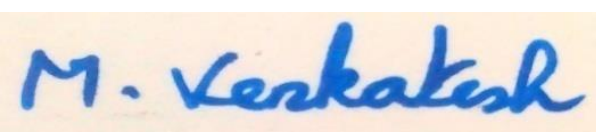

3.B. MUTHUPANDIAN

4.G.LAKSHMI PRIYA

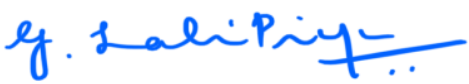

\section{$\underline{\text { Affiliation }}$}

Sethu Institute of Technology, India

REVA University, Bengaluru, Karnataka, India
Sethu Institute of Technology, India

VIT University, Chennai, TamilNadu, India 


\section{REFERENCES}

1. T. Ashley et al., "High-performance InSb based quantum well field effect transistors for low-power dissipation applications," Tech. Dig. - Int. Electron Devices Meet. IEDM, pp. 849-852, 2009.

2. T. Ashley,L. Buckle, S. Datta ,M.T. Emeny, D.G. Hayes, K.P. Hilton, R. Jefferies,T. Martin, "Heterogeneous InSb quantum well transistors on silicon for ultra-high speed, low power logic applications," IEEE Electron. Lett., vol. 43, no. 14, 2007.

3. T. Venish Kumar, N.B. Balamurugan, "Analytical modeling of InSb/AlInSb heterostructure dual gate high electron mobility transistors", AEU - International Journal of Electronics and Communications, Volume 94,2018,Pages 19-25.

4. Hiroyuki Sakaki "Velocity-Modulation Transistor (VMT) -A New Field-Effect Transistor Concept" Japanese Journal of Applied Physics, Volume 21, Number 6A, 1982.

5. Venish Kumar, T., Balamurugan, N.B. Three-dimensional analytical modeling for smallgeometry AlInSb/AlSb/InSb double-gate high-electron-mobility transistors (DGHEMTs). J Comput Electron 19, 1107-1115 (2020). https://doi.org/10.1007/s10825-02001498-2.

6. Ashley, T, Dean, AB, Elliott, CT, Jefferies, R, Khaleque, F \& Phillips, TJ 2002, 'Highspeed, low-power InSb transistors', International Electron Devices Meeting (IEDM), pp. 751-754.

7. Ashley, T, Buckle, L, Datta, S, Emeny, MT, Hayes, DG, Hilton, KP, Jefferies, R, Martin, T, hillips, TJ, Wallis, DJ, Wilding, PJ \& Chau, R 2007, 'Heterogeneous InSb quantum well transistors on silicon for ultra-high speed, low power logic applications', Electronics Letters, vol. 43, no. 14, P. 777.

8. Ashley, T, Emeny, MT, Hayes, DG, Hilton, KP, Jefferies, R, MacLean, JO, Smith, SJ, Tang, AWH, Wallis, DJ \& Webber, P 2009, 'High-performance InSb based quantum well field effect transistors for low-power dissipation applications', Technical Digest International Electron Devices Meeting, IEDM, pp. 849-852.

9. Awano, Y, Kosugi, M, Kosemura, K, Mimura, T \& Abe, M 1989, 'Short-channel effects in subquarter-micrometer-gate HEMT's: Simulation and experiment', IEEE Transactions on Electron Devices, vol. 36, no. 10, pp. 2260-2266.

10. Drummond, TJ, Morkoc, H, Lee, K \&Shur, M 1982, 'Model for modulation doped field effect transistor', IEEE Electron Device Letters, vol. 3, no. 11, pp. 338-341.

11. Kola, S, Golio, JM \& Maracas, GN, 1988, 'An analytical expression for fermi level versus sheet carrier concentration for HEMT modeling', IEEE Electron Device Letters, vol. 9, no. 3, pp. 136-138.

12. DasGupta, N \&DasGupta, A 1993, 'An analytical expression for sheet carrier concentration vs gate voltage for HEMT modelling', Solid State Electronics, vol. 36, no. 2, pp. 201-203. 
13. Lenka, TR, \& Panda, AK 2011, 'Characteristics study of 2DEG transport properties of $\mathrm{AlGaN} / \mathrm{GaN}$ and $\mathrm{AlGaAs} / \mathrm{GaAs}$-based HEMT', Semiconductors, vol. 45, no. 5, pp. 650-656.

14. A. Chakrabarty, A. K. Panda and R. Swain, "Surface Potential based modeling of Sheet Charge Density and Estimation of Critical Barrier Thickness in AlGaN/GaN HEMT," 2019 IEEE 16th India Council International Conference (INDICON), Rajkot, India, 2019, pp. 1-4, doi: 10.1109/INDICON47234.2019.9030295.

15. Parveen, S. Supriya, J. Jogi and D. Gupta, "A novel analytical model for small signal parameter for Separate Gate InAlAs/InGaAs DG-HEMT," TENCON 2012 IEEE Region 10 Conference, 2012, pp. 1-6, doi: 10.1109/TENCON.2012.6412183.

16. Amarnath, G, Panda, DK, Lenka, TR. Microwave frequency small-signal equivalent circuit parameter extraction for AlInN/GaN MOSHEMT. Int J RF MicrowComput Aided Eng. 2018;28:e21179. https://doi.org/10.1002/mmce.21179.

17. Gassoumi, M., Helali, A., Gassoumi, M. et al. High Frequency Analysis and SmallSignal Modeling of AlGaN/GaN HEMTs with SiO2/SiN Passivation. Silicon 11, 557562 (2019). https://doi.org/10.1007/s12633-018-9767-6.

18. Lee, K, Shur, Mi, Drummond, TJ \&Morkoç, H 1983, 'Electron density of the twodimensional electron gas in modulation doped layers', Journal of Applied Physics, vol. 54, no. 4, pp. 2093-2096.

19. Pu, J., Sun, J. \& Zhang, D. An accurate polynomial-based analytical charge controlmodelforAlGaN/GaNHEMT. Semiconductors 45, 1205 (2011).https://doi.org/10.1134/S1063782611090107.

20. Rathi, S, Jogi, J, Gupta, M \& Gupta, R 2009, 'Modeling of hetero-interface potential and threshold voltage for tied and separate nanoscale InAlAs-InGaAs symmetric double-gate HEMT', Microelectronics Reliability, vol. 49, no. 12, pp. 1508-1514.

21. Rathi, S, Jogi, J, Gupta, M \& Gupta, R 2010, 'An analytical charge-based drain current model for nano-scale $\mathrm{In}_{0.52} \mathrm{Al}_{0.48} \mathrm{As}-\mathrm{In}_{0.53} \mathrm{Ga}_{0.47}$ as a separated double-gate HEMT', Semiconductor Science and Technology, vol. 25, no. 11.

22. Gupta, R, Kranti, A, Haldar, S, Gupta, M \& Gupta, R 2002, 'An analytical parasitic resistance dependent Id-Vd model for planar doped InAlAs/InGaAs/InP HEMT using non-linear charge control analysis', Microelectronic Engineering, vol. 60, no. 3-4, pp. 323-337.

23. Cazaux, JL, Ng, GI, Pavlidis, D\& Chau, H 1988, 'An analytical approach to the capacitance-voltage characteristics of double-heterojunction HEMTs', IEEE Transactions on Electron Devices, vol. 35, no. 8, pp. 1223-1231. 